\title{
Association between the TACC3 rs798766 Polymorphism and Risk of Urinary Bladder Cancer: A Synthesis Based on Current Evidence
}

\author{
Xiang-Yu Meng, ${ }^{1}$ Ming-Jun Shi, ${ }^{2,3}$ Jia-Feng Chen, ${ }^{1}$ Yi Liao, ${ }^{4}$ Bang-Wang Hu, ${ }^{1}$ and \\ Ahmed Hireche ${ }^{2,3}$ \\ ${ }^{1}$ Center for Evidence-Based and Translational Medicine, Zhongnan Hospital of Wuhan University, Wuhan, China \\ ${ }^{2}$ Institut Curie, PSL Research University, CNRS, UMR 144, 75005 Paris, France \\ ${ }^{3}$ Université Paris Sud, Université Paris-Saclay, CNRS, UMR 144, 91405 Orsay, France \\ ${ }^{4}$ Department of Oncology, Nanfang Hospital, Southern Medical University, Guangzhou, China
}

Correspondence should be addressed to Xiang-Yu Meng; mengxy_whu@163.com

Received 7 February 2017; Accepted 30 April 2017; Published 5 June 2017

Academic Editor: Paola Gazzaniga

Copyright (C) 2017 Xiang-Yu Meng et al. This is an open access article distributed under the Creative Commons Attribution License, which permits unrestricted use, distribution, and reproduction in any medium, provided the original work is properly cited.

Background. A possible association between the TACC3 rs798766 polymorphism and urinary bladder cancer risk has been indicated in published literature. We performed this meta-analysis as a synthesis of all relevant data to summarize currently available evidence and to provide estimation with increased precision. Methods. EMBASE, PubMed, Google Scholar, and Wanfang Data were searched. "rs798766" and "urinary bladder cancer" were used as the search terms. A total of 6 eligible studies were identified, in which 8194 cases and 50,165 controls were investigated. Meta-analysis was performed using extracted data. Subgroup analysis by ethnicity was also performed. Population attributable risk (PAR) was calculated. Results. We found a significant association between $\mathrm{rs} 798766[\mathrm{~T}]$ and increased risk of bladder cancer, allelic[T] $\mathrm{OR}=1.27,95 \% \mathrm{CI}=1.20-1.33$. Subgroup analysis by ethnicity revealed similar results, allelic[T] $\mathrm{OR}=1.24,95 \% \mathrm{CI}=1.17-1.32$ in Caucasian subjects and allelic[T] OR $=1.33,95 \% \mathrm{CI}=1.21-1.46$ in Asian subjects. PAR based on pooled allelic ORs and the frequency of the risk allele in control subjects was $4.63 \%$ in the overall population and $3.92 \%$ in Asians and $4.36 \%$ in Caucasians. Conclusion. rs 798766 is associated with increased risk of bladder cancer, and no ethnic difference was found.

\section{Introduction}

Urinary bladder cancer (UBC) is one of the most common malignancies worldwide [1]. Clinical epidemiological studies have identified many risk factors correlated with the development of UBC, among which tobacco smoking and exposure to certain carcinogens are considered most significant $[2,3]$. Interestingly, not all exposed individuals develop UBC, suggesting that individual features, possibly genetic susceptibility, may also take part in the carcinogenesis of UBC.

As revealed in some genome-wide association studies (GWAS) [4-7], which were conducted exclusively in Caucasian subjects, dozens of single-nucleotide polymorphisms (SNPs) were identified as being associated with risk of UBC. And among which, five SNPs had also been subsequently validated in Asians, that is, rs9642880 and rs2294008 on chromosome 8q24, rs710521 on 3q28, rs2853669 on $5 \mathrm{p} 15.33$, and rs798766 on $4 \mathrm{p} 16.3$.

The rs798766 polymorphism is one of the latest found biallelic marker of $\mathrm{C} / \mathrm{T}$ variation, and the $\mathrm{C}$ is the common allele while the $\mathrm{T}$ is the risk allele. It is located in the 5th intron of TACC $3,70 \mathrm{~kb}$ away from FGFR3. Both the two genes, TACC3 and FGFR3, were reported to have an important role in the transformation of $\operatorname{UBC}[8,9]$. And the long-range regulation of a SNP to an adjacent gene was also possible [10]. Thus, it is rational to associate rs798766 with UBC both in clinical investigations and in a hypothetical mechanism. As considerable evidence is available but no synthesis has been performed, we conducted the present study, a meta-analysis aimed to summarize 
TABLE 1: Basic characteristics of included studies.

\begin{tabular}{|c|c|c|c|c|c|c|c|c|c|c|c|}
\hline First author & Year & Study design & $\begin{array}{l}\text { Genotyping } \\
\text { method }\end{array}$ & $\begin{array}{c}\text { Case } \\
(n)\end{array}$ & $\begin{array}{c}\text { Control } \\
(n)\end{array}$ & $\begin{array}{l}\mathrm{T} \text { allele Fre } \\
\text { (ctrl) }\end{array}$ & HWE & $\begin{array}{l}\text { Allelic[T] } \\
\text { OR }\end{array}$ & $\begin{array}{l}95 \% \\
\text { CILL }\end{array}$ & $\begin{array}{c}95 \% \\
\text { CIUL }\end{array}$ & Race \\
\hline Wu [7] & 2010 & $\begin{array}{l}\text { Synthesis of } 11 \text { case- } \\
\text { control sets }\end{array}$ & $\begin{array}{c}\text { SNP array, } \\
\text { PCR }\end{array}$ & 4580 & 45269 & 0.19 & In & 1.24 & 1.17 & 1.32 & Caucasian \\
\hline Figueroa [16] & 2011 & Case-control & TaqMan & 815 & 1141 & 0.118 & In & 1.32 & 1.09 & 1.59 & Asian \\
\hline Ma [17] & 2012 & Case-control & iPLEX & 176 & 959 & 0.11 & In & 1.64 & 1.19 & 2.27 & Asian \\
\hline Wang [19] & 2013 & Case-control & TaqMan & 1210 & 1008 & 0.11 & In & 1.31 & 1.09 & 1.57 & Asian \\
\hline Wang [20] & 2014 & Case-control & TaqMan & 1050 & 1404 & 0.12 & In & 1.24 & 1.05 & 1.46 & Asian \\
\hline Wang [21] & 2015 & Case-control & TaqMan & 363 & 384 & 0.11 & In & 1.53 & 1.13 & 2.08 & Asian \\
\hline
\end{tabular}

Fre (ctrl): risk allele frequency among control subjects; HWE: Hardy-Weinberg Equilibrium; OR: odds ratio; CILL: confidence interval lower limit; CIUL: confidence interval upper limit.

currently available evidence and to provide estimation with increased precision, in terms of the association between rs798766[T] and risk of UBC.

\section{Methods}

2.1. Search Strategy. A comprehensive literature search was conducted in EMBASE, PubMed, Google Scholar, and Wanfang Data up to January 2016. "rs798766" and "urinary bladder cancer" were used as the search terms. No limitations of publication language were defined. A reference list of retrieved articles was checked for potential relevant publications.

2.2. Selection Criteria. Eligible studies were selected according to the following criteria: (a) the association between rs798766 polymorphism and bladder cancer was evaluated; (b) the study has a case-control design; and (c) adjusted allelic odds ratios (ORs) and corresponding 95\% confidence intervals (CIs) were provided. The study with more information was considered where multiple reports based on overlapped population were identified. Reviews, editorials, comments, or animal studies were excluded.

2.3. Data Extraction. Two investigators independently extracted data from the eligible studies. Collected information included the following: name of the first author, year of publication, race of subjects (Caucasian or Asian), genotyping method, total number of cases and controls, frequency of risk allele in controls, Hardy-Weinberg Equilibrium (HWE) status in both cases and controls, study design, and values of ORs and 95\% CIs.

2.4. Statistical Analysis. Allelic ORs and upper and lower limits of corresponding 95\% CIs were transformed to the logarithmic scale, and the standard error of natural logarithmic OR (logOR) was calculated as se $\log \mathrm{OR}=\ln \left(\mathrm{UL}_{\mathrm{OR}}\right)-\ln \left(\mathrm{LL}_{\mathrm{OR}}\right) / 2 \times 1.96$, with $\mathrm{UL}_{\mathrm{OR}}$ and $\mathrm{LL}_{\mathrm{OR}}$ representing the upper and lower limits of the $95 \%$ confidence interval. Between-study heterogeneity was evaluated using Cochran's $Q$ test and the $I^{2}$ statistic. No significant heterogeneity was assumed if the $P$ value of Cochran's $Q$ test was not less than 0.1 and $I^{2}$ statistic not bigger than $50 \%$ [11], and a fixed-effects model assuming a common overall effect size would be used [12], according to which the pooled $\log \mathrm{OR}$ was calculated as the mean based on individual logORs weighted by corresponding variance, that is, the squared selogOR; otherwise, a random-effects model with DerSimonian-Laird estimator for tau-squared would be considered [13]. Subgroup analysis stratified by ethnicity of subjects with similar approach was performed. To test whether there exists a significant difference between the pooled results of Asians and Caucasians, a Z-test was performed. A forest plot was drawn as a visualization of meta-analysis. Begg's funnel plot and Egger's linear regression test were used to examine publication bias $[14,15]$. A $P$ value less than 0.05 was considered significant.

Risk allele frequency (RAF) among all the controls for Asian, Caucasian, and overall subjects were calculated as the average weighted by control sample size of individual studies, that is, pooled $\mathrm{RAF}_{\text {control }}=\sum_{1}^{k} \mathrm{RAF}_{i} \times n_{i} / \sum_{1}^{k} n i$, with $k$ indicating the number of all the included studies and $\mathrm{RAF}_{i}$ and $n_{i}$ representing the RAF and sample size of the control group in the $i$ th individual study. Estimated population attributable risk (PAR\%) for rs798766[T] was calculated as $\mathrm{PAR} \%=$ pooled $\mathrm{RAF}_{\text {control }} \times\left(\mathrm{OR}_{\text {pooled }}-1\right) /$ pooled $\mathrm{RAF}_{\text {control }} \times$ $\left(\mathrm{OR}_{\text {pooled }}-1\right)+1 \times 100 \%$, with pooled $\mathrm{RAF}_{\text {control }}$ representing the frequency of the risk allele in control subjects and the $\mathrm{OR}_{\text {pooled }}$ the pooled allelic odds ratio.

All the statistical analyses were performed using R 3.1.2 and the analysis package meta.

\section{Results}

3.1. Study Selection and Characteristics of Included Studies. Eight studies were retrieved after initial search [4, 16-22]. The full texts of all the eight studies were reviewed, and finally, 6 studies $[4,17-21]$ met all the selection criteria and 16 independent case-control sample sets containing 8194 cases and 50,165 controls were included in the present study.

The basic characteristics of all the included studies are shown in Table 1. No deviation from HWE was detected in any of the studies.

3.2. Quantitative Synthesis. No significant heterogeneity was detected for both the overall meta-analysis and subgroup analysis $\left(I^{2}=0, P>0.1\right.$ for both), and a fixed-effects model was used. We found a significant association between the frequency of rs798766[T] and increased risk of UBC, with allelic[T] $\mathrm{OR}=1.27$ and $95 \% \mathrm{CI}=1.20-1.33$. Subgroup analysis by ethnicity revealed similar results, with allelic[T] $\mathrm{OR}=1.24$ and $95 \% \mathrm{CI}=1.17-1.32$ in Caucasian subjects 


\begin{tabular}{|c|c|c|c|c|c|c|c|}
\hline Study & $\mathrm{TE}$ & seTE & Odds ratio & OR & $95 \% \mathrm{CI}$ & $W$ (fixed) & $W$ (random) \\
\hline \multicolumn{8}{|l|}{ Race $=$ Asian } \\
\hline M. Wang 2010 & 0.28 & 0.0963 & +1 & 1.32 & $(1.10 ; 1.60)$ & $7.5 \%$ & $7.5 \%$ \\
\hline Z. Ma 2011 & 0.49 & 0.1648 & & 1.63 & $(1.18 ; 2.25)$ & $2.6 \%$ & $2.6 \%$ \\
\hline P. Wang 2013 & 0.27 & 0.0931 & $\frac{1}{1}$ & 1.31 & $(1.09 ; 1.57)$ & $8.0 \%$ & $8.0 \%$ \\
\hline M. Wang 2014 & 0.22 & 0.0841 & \begin{tabular}{cc}
1 \\
\hdashline 1
\end{tabular} & 1.25 & $(1.06 ; 1.47)$ & $9.8 \%$ & $9.8 \%$ \\
\hline Z. Qiao 2015 & 0.43 & 0.1557 & $\begin{array}{c}1 \\
1 \\
1\end{array}$ & 1.54 & $(1.13 ; 2.09)$ & $2.9 \%$ & $2.9 \%$ \\
\hline Fixed effect model & & & $e_{1}^{1}$ & 1.34 & $(1.22 ; 1.47)$ & $30.7 \%$ & - \\
\hline \multicolumn{8}{|c|}{ Heterogeneity: $I^{2}=0 \%, \tau^{2}=0, P=0.5328$} \\
\hline \multicolumn{8}{|l|}{ Race $=$ Caucasian } \\
\hline Netherland GWAS group & 0.17 & 0.0617 & $\begin{array}{ll}1 \\
+1\end{array}$ & 1.19 & $(1.05 ; 1.34)$ & $18.2 \%$ & $18.2 \%$ \\
\hline Iceland GWAS group & 0.23 & 0.0728 & \begin{tabular}{c}
1 \\
\hdashline 1
\end{tabular} & 1.26 & $(1.09 ; 1.45)$ & $13.1 \%$ & $13.1 \%$ \\
\hline Belgium group & 0.06 & 0.1632 & 1 & 1.06 & $(0.77 ; 1.46)$ & $2.6 \%$ & $2.6 \%$ \\
\hline Germany group & 0.26 & 0.1685 & 14 & 1.30 & $(0.93 ; 1.80)$ & $2.4 \%$ & $2.4 \%$ \\
\hline Eastern Europe group & 0.44 & 0.1350 & 1 & 1.55 & $(1.19 ; 2.02)$ & $3.8 \%$ & $3.8 \%$ \\
\hline Italy, Brescia Group & -0.01 & 0.1896 & & 0.99 & $(0.68 ; 1.44)$ & $1.9 \%$ & $1.9 \%$ \\
\hline Italy, Torino Group & 0.20 & 0.1309 & & 1.22 & $(0.95 ; 1.58)$ & $4.0 \%$ & $4.0 \%$ \\
\hline Spain group & 0.30 & 0.1250 & 1 & 1.35 & $(1.06 ; 1.72)$ & $4.4 \%$ & $4.4 \%$ \\
\hline Sweden group & 0.34 & 0.1027 & $\frac{1}{1}$ & 1.40 & $(1.15 ; 1.72)$ & $6.6 \%$ & $6.6 \%$ \\
\hline UK Group & 0.16 & 0.1061 & -1 & 1.17 & $(0.95 ; 1.44)$ & $6.2 \%$ & $6.2 \%$ \\
\hline Netherland group & 0.16 & 0.1079 & $-\frac{1}{1}$ & 1.17 & $(0.95 ; 1.45)$ & $6.0 \%$ & $6.0 \%$ \\
\hline Fixed effect model & & & $\infty$ & 1.24 & $(1.16 ; 1.32)$ & $69.3 \%$ & - \\
\hline Random effects model & & & $\phi_{1}$ & 1.24 & $(1.16 ; 1.32)$ & - & $69.3 \%$ \\
\hline \multicolumn{3}{|c|}{ Heterogeneity: $I^{2}=0 \%, \tau^{2}=0, P=0.6024$} & 1 & & & & \\
\hline \multicolumn{3}{|l|}{ Fixed-effect model } & 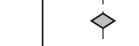 & \multirow{4}{*}{$\begin{array}{l}1.27 \\
1.27\end{array}$} & $(1.20 ; 1.34)$ & $100 \%$ & \multirow{4}{*}{$\begin{array}{c}- \\
100 \%\end{array}$} \\
\hline \multirow{3}{*}{\multicolumn{3}{|c|}{$\begin{array}{l}\text { Random-effects model } \\
\text { Heterogeneity: } I^{2}=0 \%, \tau^{2}=0, P=0.6016 \\
\text { Test for subgroup differences (fixed effect): } P=0.2079\end{array}$}} & $\diamond$ & & $(1.20 ; 1.34)$ & - & \\
\hline & & & & & & & \\
\hline & & & 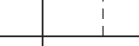 & & & & \\
\hline & & $\Gamma$ & 1 & & & & \\
\hline & & 0.5 & 1 & & & & \\
\hline
\end{tabular}

FiguRE 1: Forest plot showing meta-analysis based on 16 independent case-control sample sets. Subgroup analysis stratified by race.

and allelic[T] $\mathrm{OR}=1.33$ and $95 \% \mathrm{CI}=1.21-1.46$ in Asian subjects. The forest plot is shown in Figure 1.

The result of $Z$-test for testing the difference between Asians and Caucasians revealed no significant ethnical difference in terms of the pooled allelic ORs $(P=0.21)$.

3.3. Publication Bias. Since Begg's funnel plot was symmetric (Figure 2) and Egger's test was nonsignificant $(P=0.37)$, no evidence of publication bias was found.

3.4. RAF among Controls and PAR. As shown in Table 2, the pooled RAFs in controls were $0.12,0.19$, and 0.18 for Asians, Caucasians, and overall population, respectively. The PAR\% based on pooled allelic ORs and the frequency of the risk allele rs798766[T] in control subjects was $4.63 \%$ in the overall population and 3.92\% in Asians and $4.36 \%$ in Caucasians.

\section{Discussion}

Diseases, particularly complex ones such as UBC, have been considered as a consequence of the interaction between external risk factors and intrinsic susceptibility. In the past decades, epidemiological studies had identified many external risk factors as contributory to the carcinogenesis and progression of UBC [23-26]. On the other hand, GWAS studies have investigated genetic susceptibility to UBC. Thanks to those genome-wide association studies, several SNPs have been conferred significant susceptibility to urinary bladder cancer, such as rs9642880 at 8q24.21 (30 kb upstream from MYC), rs710521 at 3q28 (near TP63), rs2294008 at 8q24.2 (in PSCA), rs2736098 at 5p15.33 (in TERT), and rs798766 at $4 \mathrm{q} 16.3$ (on the 5 th intronic region of TACC3).

To the best of our knowledge, this study is the first that systematically summarized current evidence regarding rs798766 and risk of developing UBC and confirmed the positive association that the frequency of risk allele rs798766[T] would increase risk of UBC, with substantially increased reliability (no heterogeneity was found; $P$ values of the association for overall, Asians, and Caucasians were $1.81 \times 10-19,1.45 \times 10-9$, and $9.58 \times 10-12$, resp.). Besides, no ethnical difference was found with respect to this issue. The only limitation of our study is a lack of multiplicative risk assessment and cumulative effect of the focused SNP rs798766 together with other most related SNPs and even some external risk factors. This is mainly because of the design or selective result reported in included studies. Yet, it should be emphasized that those results are pretty crucial for an individual. A study exploring the associations between 7 SNPs and risk of UBC demonstrated that individuals with multiple risk alleles had a higher risk of bladder cancer, compared to those with 0-4 risk alleles of 7 variants $(P=3.166 \times 10-13)$ [19]. 


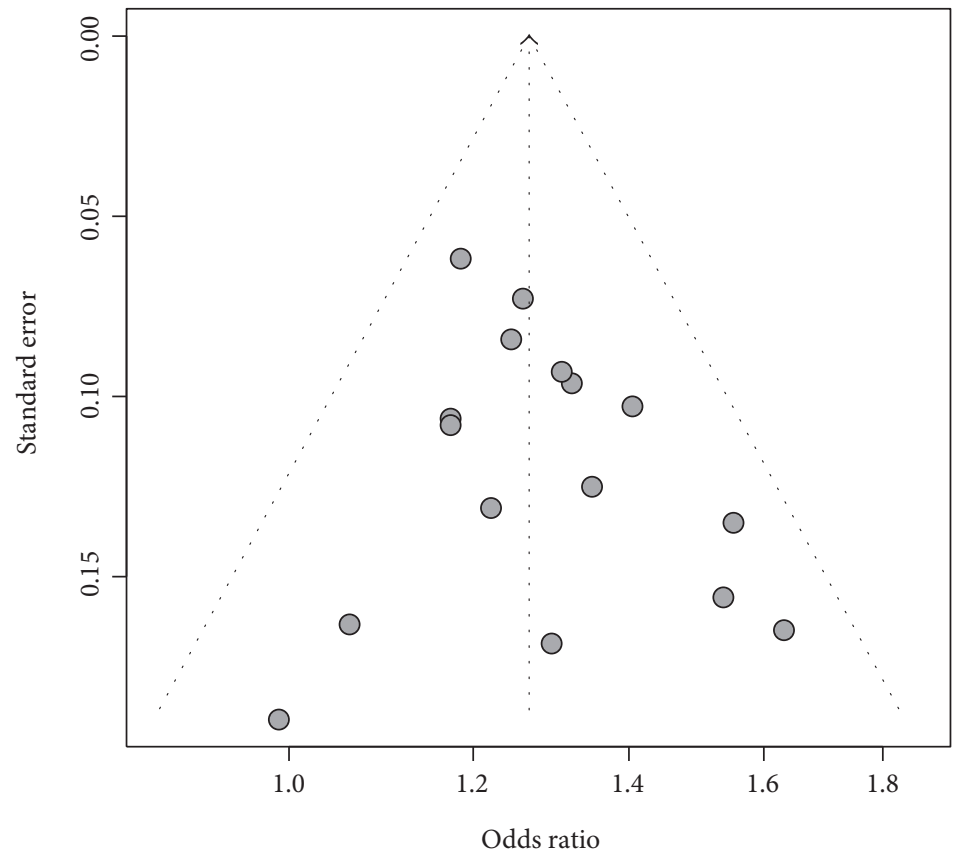

Figure 2: Funnel plot for meta-analysis based on 16 independent sample sets. No publication bias revealed.

TABLE 2: Pooled RAF in controls and PAR\%.

\begin{tabular}{lcccc}
\hline & Number of control & Pooled RAF & Pooled OR & PAR\% \\
\hline Asian & 4896 & 0.12 & 1.33 & 3.92 \\
Caucasian & 45269 & 0.19 & 1.24 & 4.36 \\
Overall & 50165 & 0.18 & 1.27 & 4.63 \\
\hline
\end{tabular}

RAF: risk allele frequency; PAR: population attributable risk.

rs798766 is located in the 5th intron of TACC $3,70 \mathrm{~kb}$ away from FGFR3. TACC3 encodes the transforming acidic coiled-coil-containing protein 3 , which is an important factor in the complex process of regulating microtubule dynamics during cell division [27]. Researchers have revealed a possible association between dysregulation of TACC3 and carcinogenesis. For instance, there are reports that upregulated TACC3 was detected in glioblastoma, non-small-cell lung cancer, non-Hodgkin's lymphoma, and multiple myeloma patients [28-31]. However, no evidence showing a possible correlation between TACC 3 and rs798766 in UBC was reported. It is also well known that the constantly activated FGFR3 by point mutation can stimulate cell proliferation and plays an oncogenic role in bladder cancer $[8,32]$. Kiemeney et al. [4] reported that the frequency of risk allele rs798766[T] in bladder Ta tumors with a high protein level of FGFR3 was 0.30 compared to 0.17 in those with a low level (OR 2.18, $P=0.073$ ), and Figueroa et al. [16] had also identified a statistically significant association between the rs798766[T] and increased FGFR3 expression both at the protein and mRNA level $(P<0.05)$. And very recently, the role of oncogenic gene FGFR3-TACC3 fusion has been discovered in UBC, in cervical cancer, in lung cancer, and in nasopharyngeal carcinoma $[9,33-35]$.

However, it should be noted that, to date, there is no evidence from functional studies exploring the potential molecular interactions among rs798766, TACC3, and FGFR3, and only very few articles proposed some plausible hypotheses. Although the association has been confirmed, the underlying mechanism has never been fully elucidated. For all of the above, we suppose that there mainly exist three possible explanations on the tight linkage between rs798766 and UBC. Firstly, we assume that the increased frequency of rs798766[T] may implicate directly to the regulatory region and enhance the promoter activity of FGFR3, triggering subsequent overexpression of FGFR3 gene which ultimately induces the transformation to tumor. Secondly, the increased frequency of rs798766[T] may accelerate the tumor formation through upregulating the expression of TACC 3 which increases the microtubule dynamic stability and massively promotes cell division, possibly enhanced by simultaneously stimulated FGFR3 activation. Thirdly, a possible role of MYC signaling pathways in UBC development has been suggested [36, 37], and interestingly, we noted that in almost all the relevant GWAS studies where a significant correlation between the rs798766[T] with bladder cancer indicated, similar findings pertaining to rs9642880[T] (30 kb upstream from MYC and exerts a long-range regulation for MYC [10]) were reported. So it seems rational to suppose that the rs798766[T] might also be involved in MYC-related pathways, manipulated by some unknown regulatory networks. Whatever the real story is, further functional studies are needed to verify these hypotheses.

In summary, this meta-analysis suggests that the TACC3 rs798766 polymorphism is significantly associated with increased risk of urinary bladder cancer. No significant ethnical difference is found in subgroup analysis. However, it is highly recommended to carry out further functional studies to clarify the mechanism behind this association. 


\section{Conflicts of Interest}

The authors declare no financial support and no conflict of interest.

\section{References}

[1] J. Ferlay, I. Soerjomataram, R. Dikshit et al., "Cancer incidence and mortality worldwide: sources, methods and major patterns in GLOBOCAN 2012," International Journal of Cancer, vol. 136, no. 5, pp. E359-E386, 2015.

[2] M. G. Cumberbatch, M. Rota, J. W. Catto, and C. La Vecchia, "The role of tobacco smoke in bladder and kidney carcinogenesis: a comparison of exposures and meta-analysis of incidence and mortality risks," European Urology, vol. 70, no. 3, pp. 458466, 2016.

[3] M. Rota, C. Bosetti, S. Boccia, P. Boffetta, and C. La Vecchia, "Occupational exposures to polycyclic aromatic hydrocarbons and respiratory and urinary tract cancers: an updated systematic review and a meta-analysis to 2014," Archives of Toxicology, vol. 88, no. 8, pp. 1479-1490, 2014.

[4] L. A. Kiemeney, P. Sulem, S. Besenbacher et al., "A sequence variant at $4 \mathrm{p} 16.3$ confers susceptibility to urinary bladder cancer," Nature Genetics, vol. 42, no. 5, pp. 415-419, 2010.

[5] L. A. Kiemeney, S. Thorlacius, P. Sulem et al., "Sequence variant on 8q24 confers susceptibility to urinary bladder cancer," Nature Genetics, vol. 40, no. 11, pp. 1307-1312, 2008.

[6] T. Rafnar, P. Sulem, S. N. Stacey et al., "Sequence variants at the TERT-CLPTM1L locus associate with many cancer types," Nature Genetics, vol. 41, no. 2, pp. 221-227, 2009.

[7] X. Wu, Y. Ye, L. A. Kiemeney et al., "Genetic variation in the prostate stem cell antigen gene PSCA confers susceptibility to urinary bladder cancer," Nature Genetics, vol. 41, no. 9, pp. 991-995, 2009.

[8] D. Cappellen, C. De Oliveira, D. Ricol et al., "Frequent activating mutations of FGFR3 in human bladder and cervix carcinomas," Nature Genetics, vol. 23, no. 1, pp. 18-20, 1999.

[9] S. V. Williams, C. D. Hurst, and M. A. Knowles, "Oncogenic FGFR3 gene fusions in bladder cancer," Human Molecular Genetics, vol. 22, no. 4, pp. 795-803, 2013.

[10] M. Wang, W. Zhang, L. Yuan, G. Fu, Q. Wei, and Z. Zhang, "Common genetic variants on $8 \mathrm{q} 24$ contribute to susceptibility to bladder cancer in a Chinese population," Carcinogenesis, vol. 30, no. 6, pp. 991-996, 2009.

[11] J. P. Higgins and S. G. Thompson, "Quantifying heterogeneity in a meta-analysis," Statistics in Medicine, vol. 21, no. 11, pp. 1539-1558, 2002.

[12] N. Mantel and W. Haenszel, "Statistical aspects of the analysis of data from retrospective studies of disease," Journal of the National Cancer Institute, vol. 22, no. 4, pp. 719-748, 1959.

[13] R. DerSimonian and N. Laird, "Meta-analysis in clinical trials," Controlled Clinical Trials, vol. 7, no. 3, pp. 177-188, 1986.

[14] C. B. Begg and M. Mazumdar, "Operating characteristics of a rank correlation test for publication bias," Biometrics, vol. 50, no. 4, pp. 1088-1101, 1994.

[15] M. Egger, G. Davey Smith, M. Schneider, and C. Minder, "Bias in meta-analysis detected by a simple, graphical test," $B M J$, vol. 315, no. 7109, pp. 629-634, 1997.

[16] J. D. Figueroa, S. Koutros, J. S. Colt et al., "Modification of occupational exposures on bladder cancer risk by common genetic polymorphisms," Journal of the National Cancer Institute, vol. 107, no. 11, 2015.

[17] Z. Ma, Q. Hu, Z. Chen et al., "Systematic evaluation of bladder cancer risk-associated single-nucleotide polymorphisms in a Chinese population," Molecular Carcinogenesis, vol. 52, no. 11, pp. 916-921, 2013.

[18] Z. Qiao, R. Wang, W. Xie, Y. Sun, C. Wu, and H. Hu, “Association between polymorphism of rs798766 and urothelial bladder cancer risk from Han population of Tianjin," Journal of Tianjin Medical University, vol. 21, no. 3, pp. 208-211, 2015.

[19] M. Wang, H. Chu, Q. Lv et al., "Cumulative effect of genomewide association study-identified genetic variants for bladder cancer," International Journal of Cancer, vol. 135, no. 11, pp. 2653-2660, 2014.

[20] M. Wang, H. Chu, F. Yan et al., "Chromosome 4p16.3 variant modify bladder cancer risk in a Chinese population," Carcinogenesis, vol. 32, no. 6, pp. 872-875, 2011.

[21] P. Wang, D. Ye, J. Guo et al., "Genetic score of multiple riskassociated single nucleotide polymorphisms is a marker for genetic susceptibility to bladder cancer," Genes, Chromosomes \& Cancer, vol. 53, no. 1, pp. 98-105, 2014.

[22] D. R. Yates, M. Roupret, S. J. Drouin et al., "Genetic polymorphisms on 8q24.1 and 4p16.3 are not linked with urothelial carcinoma of the bladder in contrast to their association with aggressive upper urinary tract tumours," World Journal of Urology, vol. 31, no. 1, pp. 53-59, 2013.

[23] F. Cantiello, A. Cicione, A. Salonia et al., "Association between metabolic syndrome, obesity, diabetes mellitus and oncological outcomes of bladder cancer: a systematic review," International Journal of Urology, vol. 22, no. 1, pp. 22-32, 2015.

[24] M. Keimling, G. Behrens, D. Schmid, C. Jochem, and M. F. Leitzmann, "The association between physical activity and bladder cancer: systematic review and meta-analysis," British Journal of Cancer, vol. 110, no. 7, pp. 1862-1870, 2014.

[25] N. Malats and F. X. Real, "Epidemiology of bladder cancer," Hematology/Oncology Clinics of North America, vol. 29, no. 2, pp. 177-189, 2015, vii.

[26] C. Xu, X. T. Zeng, T. Z. Liu et al., "Fruits and vegetables intake and risk of bladder cancer: a PRISMA-compliant systematic review and dose-response meta-analysis of prospective cohort studies," Medicine (Baltimore), vol. 94, no. 17, article e759, 2015.

[27] I. Peset and I. Vernos, "The TACC proteins: TACC-ling microtubule dynamics and centrosome function," Trends in Cell Biology, vol. 18, no. 8, pp. 379-388, 2008.

[28] C. K. Jung, J. H. Jung, G. S. Park, A. Lee, C. S. Kang, and K. Y. Lee, "Expression of transforming acidic coiled-coil containing protein 3 is a novel independent prognostic marker in nonsmall cell lung cancer," Pathology International, vol. 56, no. 9, pp. 503-509, 2006.

[29] B. C. Parker, M. J. Annala, D. E. Cogdell et al., "The tumorigenic FGFR3-TACC3 gene fusion escapes miR-99a regulation in glioblastoma," The Journal of Clinical Investigation, vol. 123, no. 2, pp. 855-865, 2013.

[30] I. H. Still, P. Vince, and J. K. Cowell, "The third member of the transforming acidic coiled coil-containing gene family, TACC3, maps in 4p16, close to translocation breakpoints in multiple myeloma, and is upregulated in various cancer cell lines," Genomics, vol. 58, no. 2, pp. 165-170, 1999.

[31] R. Yao, Y. Natsume, Y. Saiki et al., "Disruption of Tacc3 function leads to in vivo tumor regression," Oncogene, vol. 31, no. 2, pp. 135-148, 2012. 
[32] I. Bernard-Pierrot, A. Brams, C. Dunois-Larde et al., "Oncogenic properties of the mutated forms of fibroblast growth factor receptor 3b," Carcinogenesis, vol. 27, no. 4, pp. 740747, 2006.

[33] M. Capelletti, M. E. Dodge, D. Ercan et al., "Identification of recurrent FGFR3-TACC3 fusion oncogenes from lung adenocarcinoma," Clinical Cancer Research, vol. 20, no. 24, pp. 6551-6558, 2014.

[34] B. A. Carneiro, J. A. Elvin, S. D. Kamath et al., "FGFR3TACC3: a novel gene fusion in cervical cancer," Gynecologic Oncology Reports, vol. 13, pp. 53-56, 2015.

[35] L. Yuan, Z. H. Liu, Z. R. Lin, L. H. Xu, Q. Zhong, and M. S. Zeng, "Recurrent FGFR3-TACC3 fusion gene in nasopharyngeal carcinoma," Cancer Biology \& Therapy, vol. 15, no. 12, pp. 1613-1621, 2014.

[36] Y. Li, H. Liu, C. Lai, X. Du, Z. Su, and S. Gao, "The Lin28/ let-7a/c-Myc pathway plays a role in non-muscle invasive bladder cancer," Cell and Tissue Research, vol. 354, no. 2, pp. 533-541, 2013.

[37] C. W. Yeh, S. S. Huang, R. P. Lee, and B. Y. Yung, "Ras-dependent recruitment of $\mathrm{c}-\mathrm{Myc}$ for transcriptional activation of nucleophosmin/B23 in highly malignant U1 bladder cancer cells," Molecular Pharmacology, vol. 70, no. 4, pp. 14431453,2006 


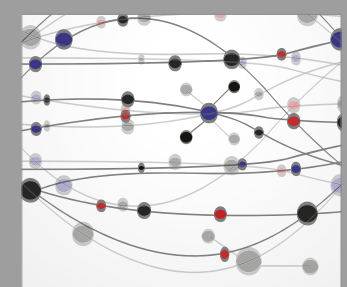

The Scientific World Journal
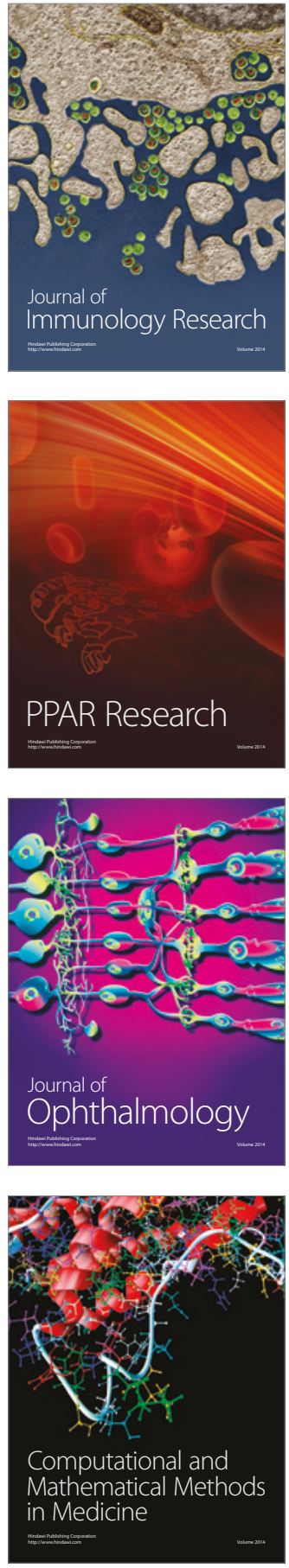

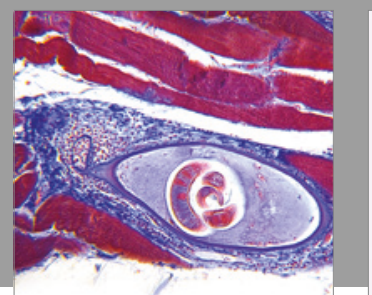

Gastroenterology Research and Practice
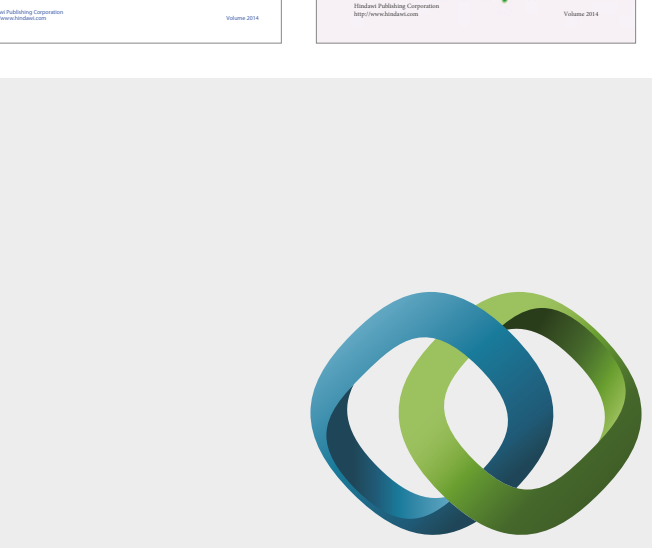

\section{Hindawi}

Submit your manuscripts at

https://www.hindawi.com
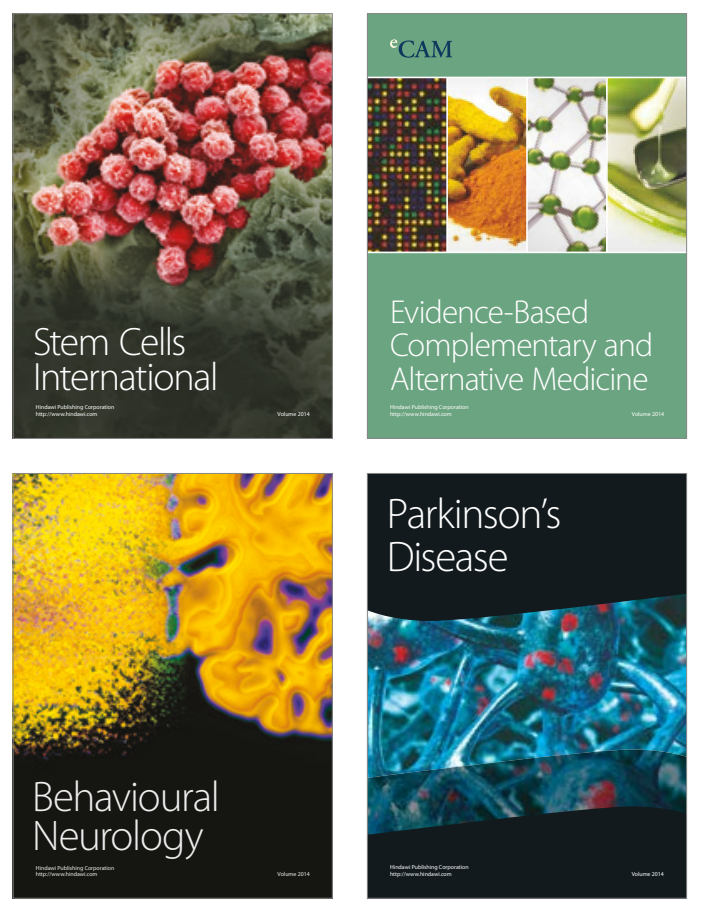
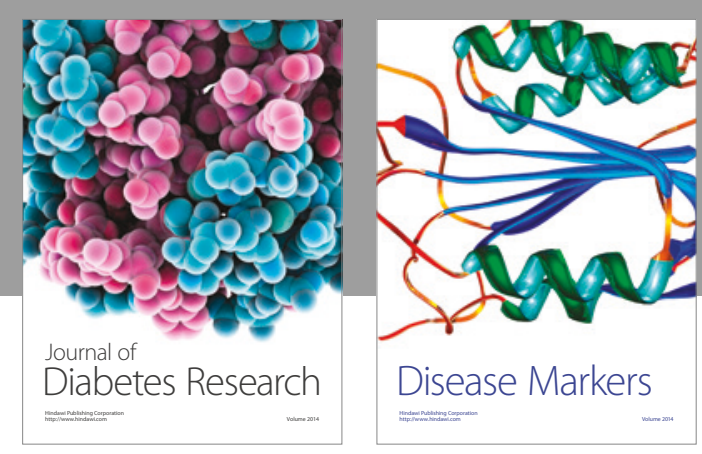

Disease Markers
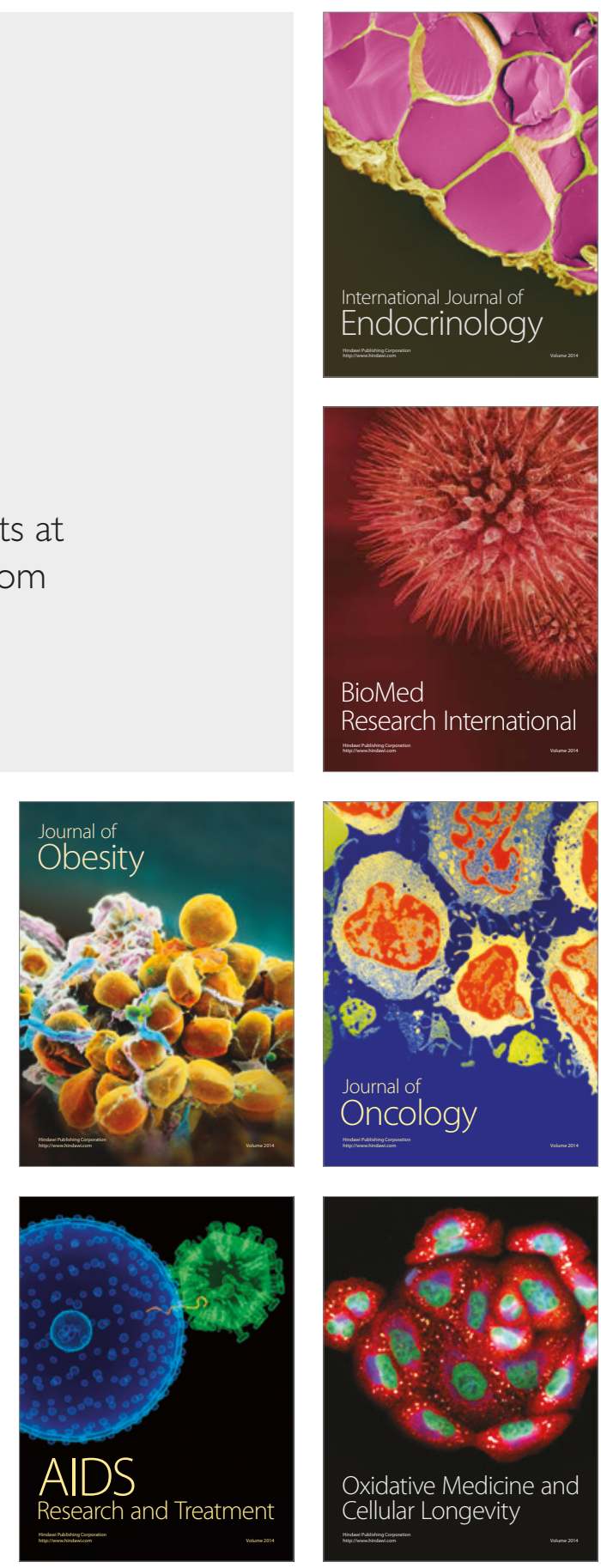